\title{
Gravity is an Important Determinant of Oxygenation during One-Lung Ventilation
}

\author{
Szegedi L1, d'Hollander A, Vermassen F², De Ryck F², Wouters P' $^{\mathbf{1}}$ \\ ${ }^{1}$ Dept. of Anesthesiology, University Hospital Gent, Belgium \\ 2 Thoracovascular Surgery , University Hospital Gent, Belgium
}

The role of gravity in the redistribution of pulmonary blood flow during one-lung ventilation (OLV) has recently been questioned. To address this controversial but clinically important issue we used an experimental approach that allowed us to differentiate the effects of gravity from the effects of hypoxic pulmonary vasoconstriction on arterial oxygenation during OLV in patients scheduled for thoracic surgery.

\section{Methods}

Forty patients with chronic obstructive pulmonary disease scheduled for right lung tumour resection, were randomized to undergo dependent (left) one-lung ventilation ( $\mathrm{D}-\mathrm{OLV} ; \mathrm{n}=20$ ) or non-dependent (right) one-lung ventilation (NDOLV ; $n=20)$ in the supine and left lateral positions. Partial pressure of arterial oxygen $\left(\mathrm{PaO}_{2}\right)$ was measured as a surrogate for ventilation/perfusion matching. Patients were studied before surgery in closed chest conditions.

Group D-OLV

(Left lung ventilated dependent lung ventilation)

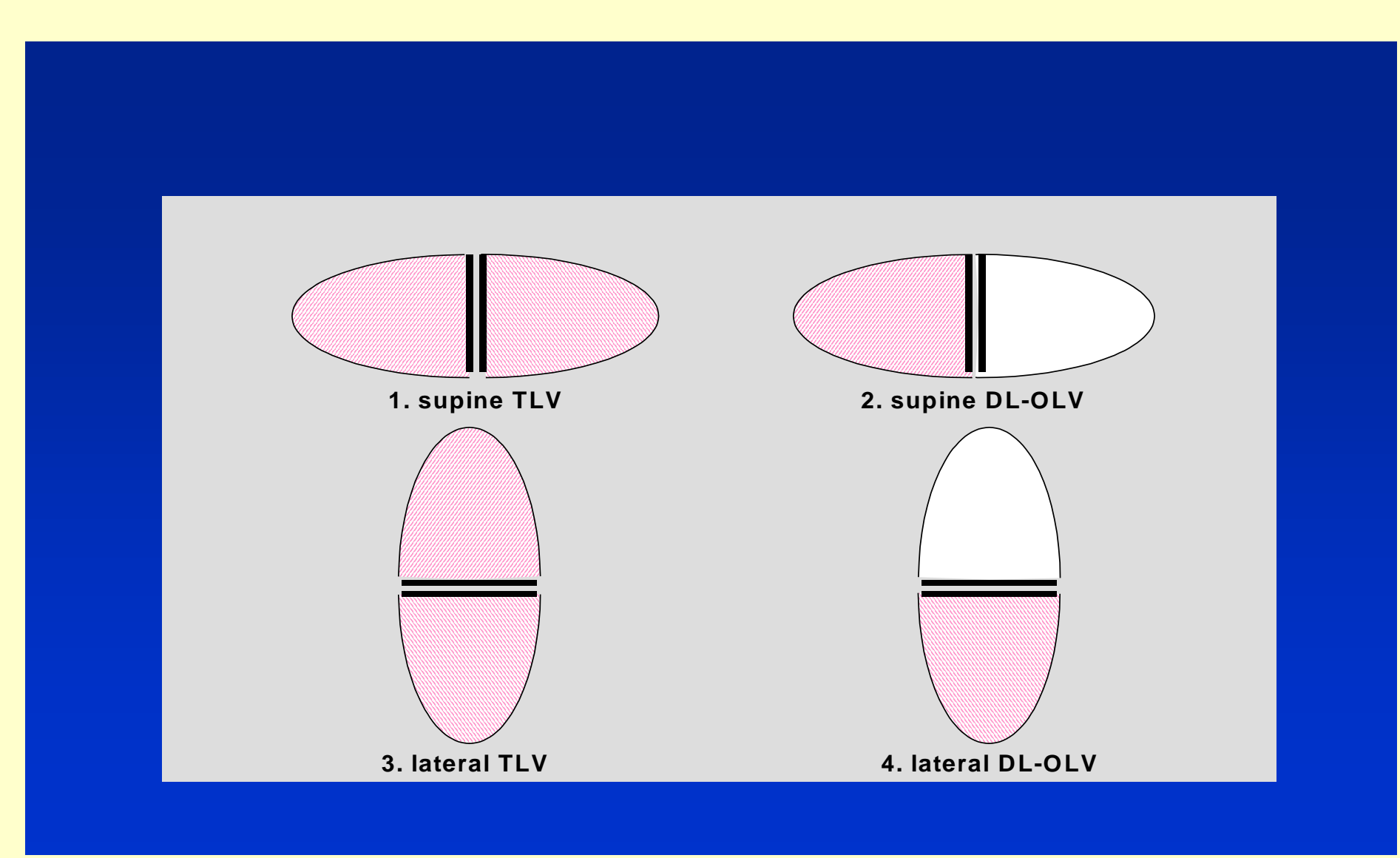

\section{Results}

When compared to bilateral lung ventilation, both D-OLV and ND-OLV caused a significant and equal decrease in $\mathrm{PaO}_{2}$ in the supine position. However, D-OLV in the lateral position was associated with a higher $\mathrm{PaO}_{2}$ as compared to the supine position [274.2 (77.6) vs. $181.9(68.3) \mathrm{mmHg}, \mathrm{p}<0.01$, ANOVA]. In contrast, in patients undergoing ND-OLV, $\mathrm{PaO}_{2}$ was always lower in the lateral as compared to the supine position [105.3 (63.2) vs. $187(63.1) \mathrm{mmHg}, \mathrm{p}<0.01$, ANOVA].

\section{Conclusion}

The relative position of the ventilated versus the nonventilated lung markedly affects arterial oxygenation during OLV. These data suggest that gravity affects ventilation-perfusion matching independently from HPV.

\section{Statistical Analysis}

Statistical analysis was performed with the SigmaPlot 10 software package, version 10.0 for Windows, Systat Software Inc. .

First, the assumption that data are sampled from populations with identical standard deviations (SD) was tested using the method of Bartlett. The assumption that the differences are sampled from populations that follow Gaussian distribution was verified using the method of Kolmogorov and Smirnov. Patient characteristics, preoperative pulmonary function results and preoperative arterial blood gas values of the 40 patients were compared with the Student's unpaired t-test. Thereafter, differences between the groups and within groups were analyzed with the One-way Analysis of Variance (ANOVA) with the Tukey-Kramer Multiple Comparisons Test.

Values of $p<0.05$ were accepted as statistically significant. Data are presented as mean \pm SD.

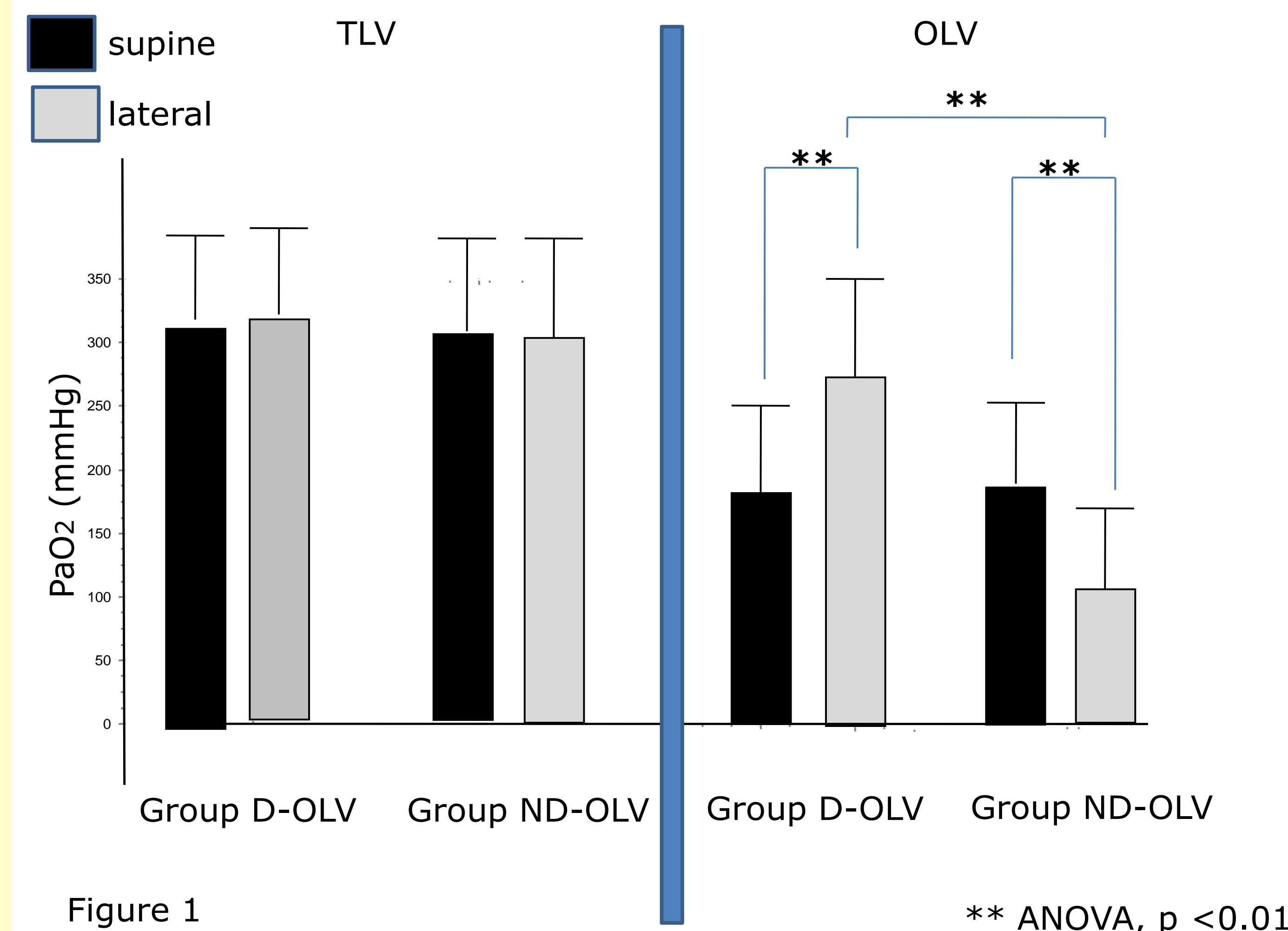

